\title{
Bacteriological profile of burn wound infections with reference to biofilm formation
}

\section{Abstract}

Purpose: Burns are one of the most common and devastating forms of trauma. A prospective cross-sectional study of bacterial isolates from wounds of patients admitted to burns unit of a tertiary care hospital was undertaken to study the bacteriological profile and biofilm formation.

Materials and methods: Sample swabs were processed by inoculation onto MacConkey and Chocolate media. Biofilm formation was detected using three methods- tissue culture plate (TCP), Tube method (TM) and Congo red agar (CRA) method.

Results: Among 100 burns patients who were studied, 90 (90\%) samples gave positive bacterial culture, Pseudomonas aeruginosa was the most common organism isolated. 42 isolates $(46.6 \%)$ were biofilm positive.

Conclusion: The commonest organism causing burn wound sepsis and forming biofilm in our study was Pseudomonas aeruginosa.

Keywords: burns, biofilm, pseudomonas aeruginosa, klebsiella species
Volume 4 Issue 4 - 2017

\author{
Asima Banu,' Presteena Mathew, ${ }^{2}$ Manasa S, ${ }^{2}$ \\ Abith B Shetty ${ }^{2}$ \\ 'Department of Microbiology, Bangalore Medical College \& \\ Research Institute, India \\ ${ }^{2}$ Bangalore Medical College \& Research Institute, India
}

\begin{abstract}
Correspondence: Asima Banu, Professor, Department of Microbiology, Bangalore Medical College \& Research Institute, Bangalore, Karnataka, India, Tel 9845720258 ,

Email asima.banu@gmail.com
\end{abstract}

Received: March 13, 2017 | Published: April 25, 2017
Abbreviations: TCP, tissue culture plate; TM, tube method; CRA, congo red agar

\section{Introduction}

Burns are one of the most common devastating forms of trauma. Burn patients are ideal hosts for opportunistic infections. ${ }^{1}$ Thermal injury causes instant coagulative necrosis, which rapidly becomes a favourable niche for bacterial colonisation and proliferation. The eschar provides a devitalised, protein rich environment, which further benefits bacterial proliferation through its exclusion from the systemic circulation and impaired local immune responses. The burn site remains relatively sterile during the first 24hour; thereafter, colonization of the wound by gram negative bacteria is common. ${ }^{2}$ Organisms originate from the patient's own skin, gut and respiratory flora, as well as from contact with contaminated health care environments and workers. ${ }^{3}$ Biofilms are complex communities of surface attached aggregates of microorganisms embedded in a selfsecreted extracellular polysaccharide matrix, or slime. ${ }^{4}$ Once formed, biofilms act as efficient barriers against antimicrobial agents and the host immune system, resulting in persistent colonization and/or infection at the site of biofilm formation. ${ }^{5}$ Bacteria within a biofilm typically undergo a phenotypic change whereby microbial virulence factor production is altered and metabolic rate and motility are reduced. Channels formed within the protective environment of the biofilm facilitate the transport of nutrients and microbial waste products. ${ }^{6}$ The survival rates for burn patients have improved substantially in the past few decades due to advances in modern medical care in specialized burn centres. Improved outcomes for severely burned patients have been attributed to medical advances in fluid resuscitation, nutritional support, pulmonary care, burn wound care, and infection control practices. $^{\text {? }}$

\section{Materials and methods}

The study was conducted in the Department of Microbiology of a tertiary care hospital after obtaining approval from Institutional Ethical Committee. Samples were taken from patients admitted with burns to burns ward of teaching hospitals attached to a Medical College. This was a prospective cross sectional study where 100 patients having burn wound infection were included in the study. Patients co-infected with HIV, Hepatitis B virus and patients having malignancy have been excluded from the study.

Samples for microbiological examination were collected from secretions adjacent to the burn wounds by sterile cotton swabs and immediately transferred to the Microbiology lab. The specimens were processed immediately first by inoculating onto media culture and then direct smear examination by Gram Stain to avoid contamination. The samples were processed by inoculating onto the following media: 5\% sheep blood agar and MacConkey agar, incubated at $37^{\circ} \mathrm{C}$ aerobically for 24 hours, Chocolate agar, incubated at $37^{\circ} \mathrm{C}$ in the presence of $5-10 \%$ of $\mathrm{CO}_{2}$ in a candle jar for 24 hours.

These were incubated for 48hours and if there was no growth, the media was incubated for 8 more days to allow the growth of slow growing or fastidious organisms. All the cultures were subjected to Gram stain and later isolates were identified using standard microbiological procedures such as catalase test, oxidase test, IMViC tests, sugar fermentation etc and tested for antimicrobial susceptibility by means of agar disc diffusion method of Kirby Bauer according to the guidelines of Clinical and Laboratory Standards Institute (2012).

Detection of biofilm formation was done by three methodsTissue culture plate method (TCP), Tube method (TM) and Congo red formation. ${ }^{8,9}$ Figure 1 methodology was adapted as according to Mathur et al. ${ }^{9}$

\section{Tissue culture plate method (TCP)}

Isolates from fresh agar plates were inoculated in TSBglu (trypticase soy broth with $1 \%$ glucose) and incubated for 18 hour at $37^{\circ} \mathrm{C}$ in stationary condition and diluted 1 in 100 with fresh medium. 
Individual wells of sterile, polystyrene, 96 well-flat bottom tissue culture plates wells were filled with $0.2 \mathrm{ml}$ aliquots of the diluted cultures and only broth served as control to check sterility and nonspecific binding of media.

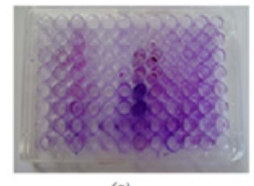

(a)
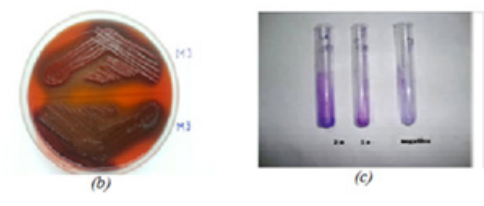

Figure I Screening for biofilm production using various methods.

a. Tissue culture plate method

b. Congo red agar method

c. Tube method

The tissue culture plates were incubated for 18 hours and 24 hours at $37^{\circ} \mathrm{C}$. After incubation content of each well was gently removed by tapping the plates. The wells were washed four times with $0.2 \mathrm{~mL}$ of phosphate buffer saline (PBS $\mathrm{pH}$ 7.2) to remove free-floating 'planktonic' bacteria. Biofilms formed by adherent 'sessile' organisms in plate were fixed with sodium acetate $(2 \%)$ and stained with crystal violet $(0.1 \% \mathrm{w} / \mathrm{v})$. Excess stain was rinsed off by thorough washing with deionized water and plates were kept for drying. Adherent cells usually formed biofilm on all side wells and were uniformly stained with crystal violet. Optical density (OD) of stained adherent bacteria were determined with a micro ELISA auto reader at wavelength of $570 \mathrm{~nm}(\mathrm{OD} 570 \mathrm{~nm})$. These OD values were considered as an index of bacteria adhering to surface and forming biofilms.

Experiment was performed in triplicate and repeated three times, the data was then averaged and standard deviation was calculated. To compensate for background absorbance, OD readings from sterile medium, fixative and dye were averaged and subtracted from all test values. The mean OD value obtained from media control well was deducted from all the test OD values. For the purpose of data calculation, Table 1 shows the classification based on OD values.

Table 1 Classification of biofilm formation by tissue culture plate assay

\begin{tabular}{lll}
\hline Mean O.D value & Adherence & Biofilm formation \\
\hline$<0.120$ & Non & Non/weak \\
$0.120-0.240$ & Moderate & Moderate \\
.0 .240 & Strong & Strong \\
\hline
\end{tabular}

\section{Tube method (TM)}

TSBglu $(10 \mathrm{~mL})$ was inoculated with loop ful of microorganism from overnight culture plates and incubated for 24 hours at $37^{\circ} \mathrm{C}$. The tubes were decanted and washed with PBS (pH 7.3) and dried. Dried tubes were stained with crystal violet $(0.1 \%)$. Excess stain was removed and tubes were washed with deionized water. Tubes were than dried in inverted position and observed for biofilm formation.

Biofilm formation was considered positive when a visible film lined the wall and bottom of the tube. Ring formation at the liquid interface was not indicative of biofilm formation. Tubes were examined and the amount of biofilm formation was scored as 0 -absent, 1-weak, 2-moderate or 3-strong Experiments were performed in triplicate and repeated three times.

\section{Congo red agar method (CRA)}

This method requires the use of a specially prepared solid medium -brain heart infusion broth (BHI) supplemented with 5\% sucrose and Congo red. The medium was composed of BHI $(37 \mathrm{gms} / \mathrm{L})$, sucrose $(50 \mathrm{gms} / \mathrm{L})$, agar no. $1(10 \mathrm{gms} / \mathrm{L})$ and congo red stain $(0.8 \mathrm{gms} / \mathrm{L})$. Congo red was prepared as concentrated aqueous solution and autoclaved at $121^{\circ} \mathrm{C}$ for 15 minutes, separately from other medium constituents and was then added when the agar had cooled to $55^{\circ} \mathrm{C}$. Plates were inoculated and incubated aerobically for 24 to 48 hours at $37^{\circ} \mathrm{C}$.

Positive result was indicated by black colonies with a dry crystalline consistency. Weak slime producers usually remained pink, though occasional darkening at the centers of colonies was observed. A darkening of the colonies with the absence of a dry crystalline colonial morphology indicated an indeterminate result. The experiment was performed in triplicate and repeated three times.

\section{Results}

Among 100 burns patients who were studied 52 were women $(52 \%)$ and 48 were men (48\%). $90(90 \%)$ samples gave positive bacterial culture, whereas $10(10 \%)$ showed no bacterial growth. Among 90 isolates 42 (46.6\%) were biofilm positive and 48(53.3\%) were biofilm negative.

Pseudomonas aeruginosa 38(42.2\%) was the common organism to be isolated and forming biofilm followed by Klebsiella species 22(24.4\%), Proteus spp. 18(20\%) and Methicillin resistant Staphylococcus aureus 12 (13.3\%) (Figure 2).

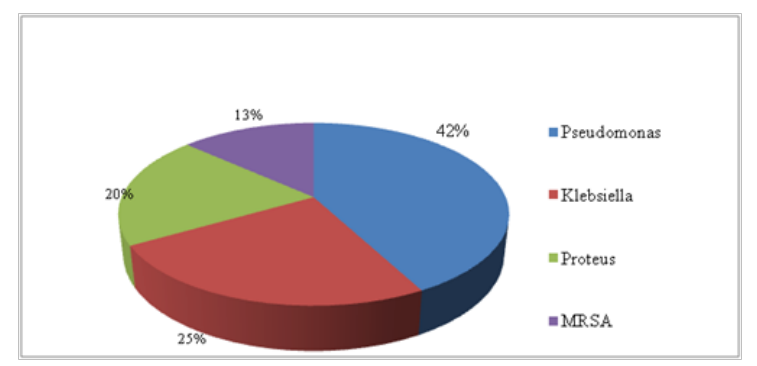

Figure 2 Pie diagram showing various organisms isolated.

\section{Discussion}

Burn wound infections are one of the most important and potentially serious complications that occur in the acute period following injury. Infections remain the leading cause of death among patients who are hospitalized for burns. The risk of burn wound infection is directly correlated to the extent of the burn and is related to impaired resistance resulting from disruption of the skin's mechanical integrity and generalized immune suppression.

In the present study females (52\%) are affected more compared to males $(48 \%)$. This may be because of the reason that accidental burns are more common in females as they tend to spend more time near fire and this results are in concordance with a study by Singh et al and the most common age group affected was $20-40 \mathrm{yrs} .^{10}$

In our study there was $90 \%$ of culture positivity with a single organism, The high percentage of positive bacterial cultures of the wound swab may be attributed to the fact that the burn wound has a much higher incidence of infections compared with other forms 
of trauma because of extensive skin barrier disruption as well as alteration of cellular and humoral immune responses. ${ }^{11}$

The most common organism isolated in our study was Pseudomonas aeruginosa $38(42.2 \%)$ followed by Klebsiella species 22(24.4\%), Proteus spp. 18(20\%) and Methicillin resistant Staphylococcus auereus $12(13.3 \%)$. There is a predominance of gram negative bacteria i.e. Pseudomonas aeruginosa in specific from the cultures. This agrees with a study by Singh et al., ${ }^{10}$ who found the most commonly isolated organisms from burn patients were Pseudomonas species followed by Staphylococcus aureus and Klebsiella species and these results are also in accordance with other study by Ozumba et al. ${ }^{12}$

Biofilms are microbial communities encased within polysaccharide rich extracellular matrix on surfaces of wounds. They are associated with drastically enhanced resistance against most antimicrobial agents leading to treatment failures. In our study Pseudomonas aeruginosa is the most common organism producing biofilm in burns patients followed by Staphyloccus aureus and Klebsiella species but this is not in concordance with a study by Madam et al., ${ }^{13}$ in which Klebsiella pneumoniae is often involved in biofilm-related infections. Biofilm producing organisms were associated with therapeutic failure and infection was resolved only on surgical debridement.

\section{Conclusion}

The gram negative bacteria are more predominant as causative agents for burn wound infections than the gram positive bacteria. The commonest organism causing burn wound sepsis and forming biofilm is Pseudomonas species. So, appropriate wound care is necessary for burn wound management. The obtained data confirm the necessity of describing an effective management scheme including debridement, irrigation and antibiotic administration.

\section{Acknowledgements}

None.

\section{Conflict of interest}

The author declares no conflict of interest.

\section{References}

1. Cochran A, Morris SE, Edelman LS, et al. Systemic Candida infection in burn patients: a case-control study of management patterns and outcomes. Surg Infect (Larchmt). 2002;3(4):367-374.

2. Pruitt BA, McManus AT, Kim SH, et al. Burn wound infections: current status. World J Surg. 1998;22(2):135-145.

3. Murray C, Hospenthal DR. Burn wound infections. E medicine. 2008.

4. Stoodley P, Sauer K, Davies DG, et al. Biofilms as complex differentiated communities. Annu Rev Microbiol. 2002;56:187-209.

5. Edwards R, Harding KG. Bacteria and wound healing. Curr Opin Infect Dis. 2004;17(2):91-96.

6. Harrison-Balestra C, Cazzaniga AL, Davis SC, et al. A wound-isolated Pseudomonas aeruginosa grows a biofilm in vitro within 10 hours and is visualized by light microscopy. Dermatol Surg. 2003;29(6):631-635.

7. Church D, Elsayed S, Reid O, et al. Burn wound infections. Clin Microbiol Rev. 2006;19(2):403-434.

8. Christensen GD, Simpson WA, Younger JJ, et al. () Adherence of coagulase-negative staphylococci to plastic tissue culture plates: a quantitative model for the adherence of staphylococci to medical devices. J Clin Microbiol. 1985;22(6):996-1006.

9. Mathur T, Singhal S, Khan S, et al. () Detection of biofilm formation among the clinical isolates of Staphylococci: an evaluation of three different screening methods. Indian J Med Microbiol. 2006;24(1):25-29.

10. Singh NP, Goyal R, Manchanda V, et al. () Changing trends in bacteriology of burns in the burns unit, Delhi, India. Burns. 2003;29(2):129-132.

11. Sanyal SC, Mokaddas EM, Gang RX, et al. Microbiology of septicaemia in burn patients. Ann Burns Fire Disasters. 1998:11(1).

12. Ozumba UC, Jiburum BC. Bacteriology of burn wounds in Enugu, Nigeria. Burns. 2000;26(2):178-180.

13. Madam M, Petersenb I, Cheastyc T. Biofilm-forming Klebsiella pneumoniae strains have greater likelihood of producing extended spectrum b-lactamases. Elsevier. 2008;10:369-371. 